\title{
Ensayo clínico con broncodilatadores en bronquitis obstructiva del lactante y preescolar menor
}

\author{
Drs. Isabel Torrealba $M_{,, *}$ Rodolfo Burdach W.,** Patricia Glasser, Lina Boza** \\ (Departamento Broncopulmonar Hospital Paula Jaraquernada)
}

\begin{abstract}
In order to evaluate the nesponse with Beta-Adrenergit stimulants in small children with Wheezy Bronchitis, we did a clinical study with Fenoterol an Metaproterenol comparing them with a control graup.

The statistical analysis of each of the elinical parameters, demonstrated significant differences only in the decrease of respiratory trequency with Beta-Adrenergic stimulants in comparison to controls.

Functional studies are necessary to confirm if the reduction in respiratory frexuency results from a decrease in air flow resistance.

Any way this study suggests that the lwnefits of Beta-Adrenergic stimulants are limited in obstructive bronchitis at this age.
\end{abstract}

La bronquitis obstructiva del lactante y preescolar menor es un cuadro clínico de importancia creciente por su alta incidencia, clyo manejo terapéutico suele presentar dificultades, por la falta de una respuesta rápida frente a los inúltiples medicamentos usados en la actualidad (antiinflamatorios, broncodilatadores, etc.).

Esto en parte se explica por los factores fisiopatologicos que cundicionan su evolución, en la cual la resistencia al thujo aéreo estaria aumertada fundamentalmente por elementos obstructivos inflamatorios, como son el edema $y$ la hipersecreción bronquial, más que el broncoespasmo.

Por otra parte debemos recordar que el pulmón del nin̄o menor presenta características anatómicas y funcionales ${ }^{10,13}$ que de por sí estín condicionando un aumento de la resistencia al flujo aéreo, como son el menor desarrollo del tejido de sostén y muscular bronquial, la mayor proporción de glándulas mucosas, y las modificaciones dinámicas que suffe la vía aérea durante los movimientos respiratorios.

* Médico bezado de Pediatria. Hosp. Paula Jararyuemada.

**Médica Jefe del Depto. Broncopulmonar. Hossp. Paula jaraquemakda.

* Méclico becarlo U. de Chile, Pediatria Hosp. Paula Jaraguemadia

** Mexhico del Jepto. Broncrpultronaz. Hospp. Pasula Jaraquethatlia.
Esto encuentra su confirmación en la literatura, 1, 5.6.9.11 en la cual destacan los estudios funcionales con técnicas oscilométricas o con pletismógrafo, hechas en lactantes y nin̄os menores, al usar broncodilatadores del tipo beta-adrenérgicos, sin lograr demostrar efecto broncodilatador. ${ }^{12}$ Sin embargo, hay experiencias clinicas, hechas en ninos menores, ${ }^{3}$ usando broncodilatadores que sugieren que estas drogas tendrian algún efecto en el tratamiento de niños menores, portadores del Sindrome Bronquial Obstructivo.

Por otra parte, el seguimiento de niños con bronquitis obstructiva actualmente pemite sugerir que el porcentaje de asmáticos tiende a aumentar en este grupo etario?

Esto motivó un estudio clínico en lactantes y preescolares menores con bronquitis obstructiva, para evaluar el efecto de los broncodilatadores beta-adrenérgicos como Fenoterol y Metaproterenol, comparados con un grupo control.

\section{MATERLAL Y METODO}

Se estudiaron 44 niños desde el mes de julio de 1979 a julio de 1980 , hospitalizados desde su ingreso con el diagnóstico de bronquitis obstructiva aguda o bronquitis obstructiva recidivante, cuyas edades tluctuaban entre los 5 meses y los 32 meses de vida. (Tablas Nos. 1 y 2 .) 
Tabla J

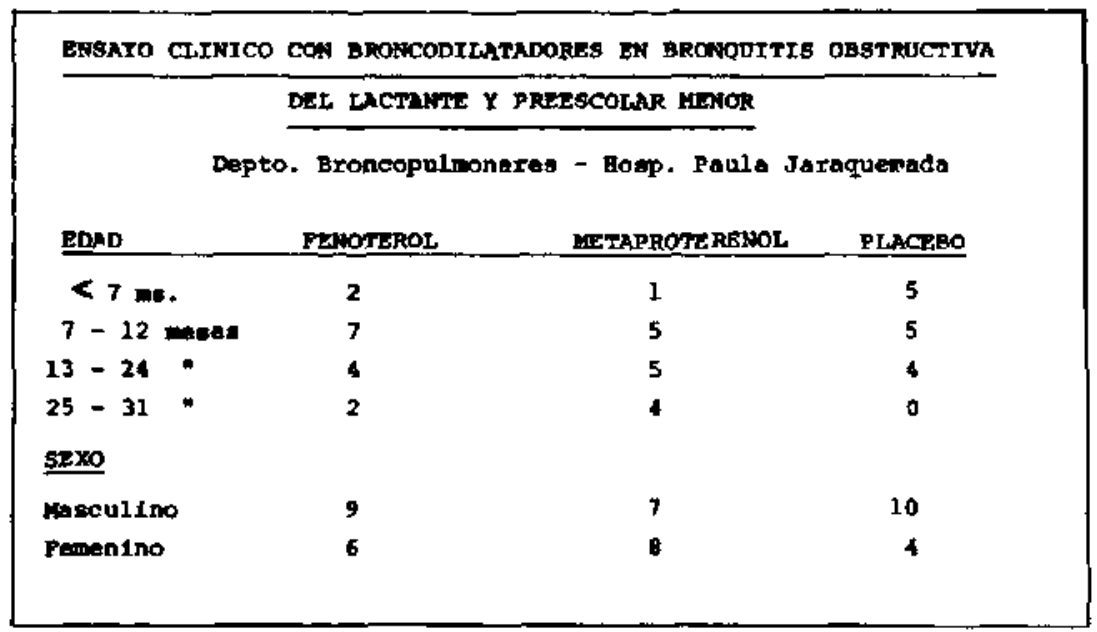

Tabla 2

ENSAXO CLINICO CON BRONCODILATADORFS FN BRONOUITIS OBSTRUCTIVA

DEL LACTANTE Y PREESCOLAP MFNOR

Depto. Broncopulmonares - Hosp. Paula Jaraquemada

\begin{tabular}{l} 
EXAMENFS \\
FENOTERNL \\
\hline
\end{tabular}

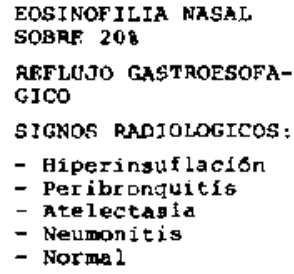

1
7
6
2
2
7
0

El diagnóstico se realizô en base a la anamnesis y examen físico. Su estudio incluyó radiografía de tórax al ingreso y al quinto día de evolución; trán-

sito E.G.D. en los casos subintrantes, y estudio de gases en sangre arterial. (Tablas Nos. 3 y 4.)

ENSAYO CLIHICO CON BRTNCODILATADORES EN RRCNOUITIS ORSTRUC-

TIVA DEL LACTANTE $\times$ PREFSCOLAR MENOA

Depto. Broncopulmonareg - Hosp. Paula Jaraquemada

ANTECEDENES FAMILIARES ATOPICOS DIRECTOS

FENOTEROL_- METAPROTENOL PLPCFBO

ANTECEDENTES PERSONALES ATOPICOS D 


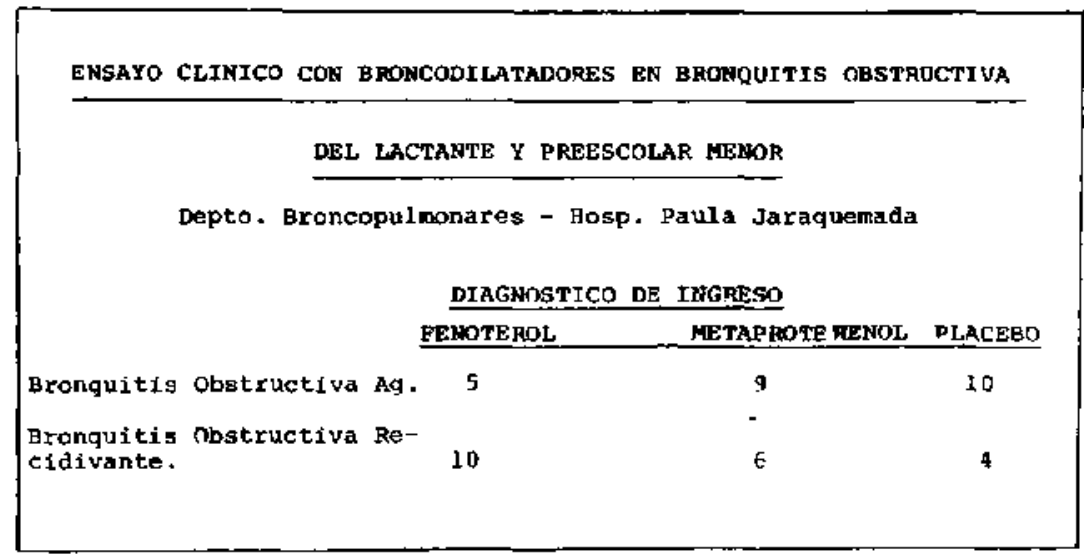

E] tratamiento consistió en Fenoterol en 15 pacientes en dosis de: 1 a 2 inhalaciones $(0.2$ a 0.4 ng) cada 8 horas por 48 horas y Fenoterol oral 1,25 $\mathrm{mg}$ ( $1 / 2$ compr) c/8 horas en menores de 2 años $y$ $2,50 \mathrm{mg}$ ( 1 comp c/8 horas por $10 \mathrm{dias}$ ) en los mayores. 15 pacientes recibieron Metaprotereno] en dosis de 1 inhalación en mayores de 2 años $(0,65 \mathrm{mg})$ cada 8 horas por 48 horas y en forma oral $0,5 \mathrm{mg} \times \mathrm{kg}$ de peso $\mathrm{c} / 8$ horas por $\mathbf{1 0}$ días.

El grupo control, constituido por 14 pacientes, recibió placébo 1 inhalación c/8 horas por 48 horas $y$ oral cada 8 horas por 10 días.

El control clínico se realizó, registrando la frecuencia respiratoria y cardiaca; se consignaron en un score de 0 a 3 los siguientes parámetros clínicos: retracción inspiratoria, espiración prolongada, sibilancias y estertores húmedos; en el que 0 equivalía a los asintomáticos: 1 a los leves con escasa retracción infracostal, sin retracción supraesternal ni intercostal, sin aleteo nasal y con buen estado general. Espiración algo prolongada, sibilancias y estertores húmedos aislados. El grado 2 era moderado con retracción infracostal, supraesternal e intercostal; aleteo nasal. Aumento del diámetro anteroposterior del tórax y compromiso del estado general. Espiración prolongada y abundantes estertores húmedos y sibilancias. Grado 3 equivalía a los severos: gran compromiso del estado general, retracción intercostal máxima o con tendencia al agotamiento y cianosis. Marcada disminución del murmullo vesicular y de los ruidos bronquiales.

Para objetivar al máximo, el control estuvo a cargo de una misma persona.

Se realizó un examen al ingreso, a las $1 / 2,6$ y a las 24 horas; luego diariamente durante los 10 días de tratamiento.

\section{RESULTADOS}

Se analizó estadísticamente cada parámetro por separado, comparíndolo con el placebo. (Ver gráficos 1 y 2.)

Gräfico l

\section{ENSAYOCLINICO CON BRONCODILATADORES EN BRONQUITIS OBSTRUCTIVA DEL LACTANTE Y PRE-ESCOLAR MENOR.}

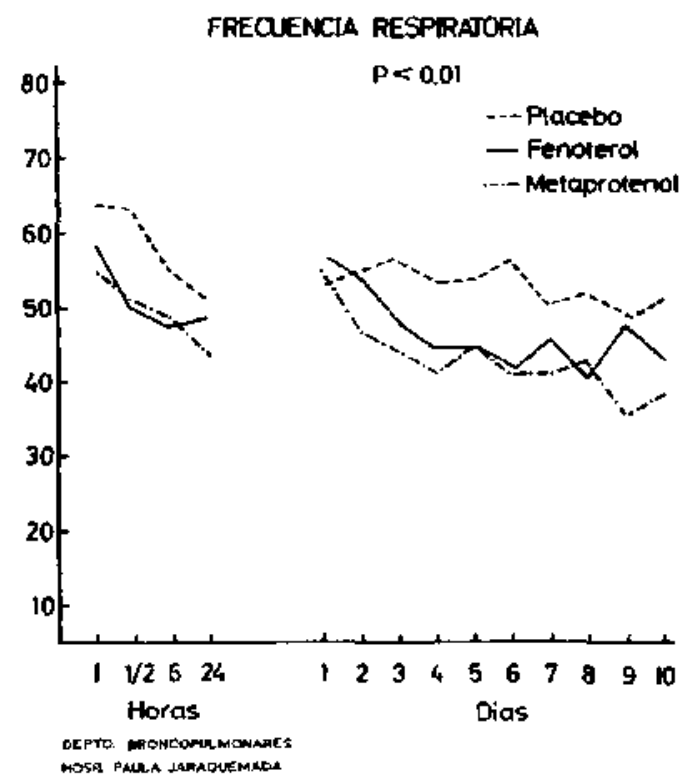


Giráfico 2

\section{ENSAYO CLINICO CON \\ BRONCODILATADORES EN BRONQUITIS \\ OBSTRUCTIVA DEL LACTANTE Y \\ PRE-ESCOLAR MENOR.}

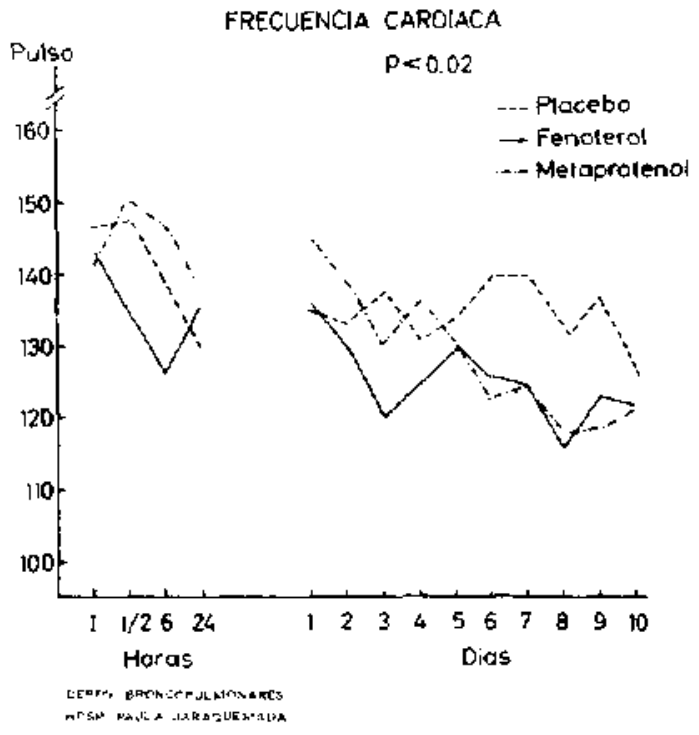

Gíráfico 3

\section{ENSAYO CLINICO CON}

BRONCODILATADORES EN BRONQUITIS

OBSTRUCTIVA DEL LACTANTE Y PRE-ESCOLAR MENOR.

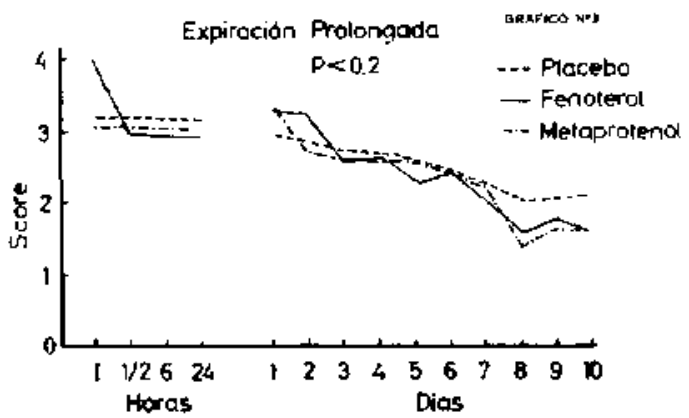

Grafice 4

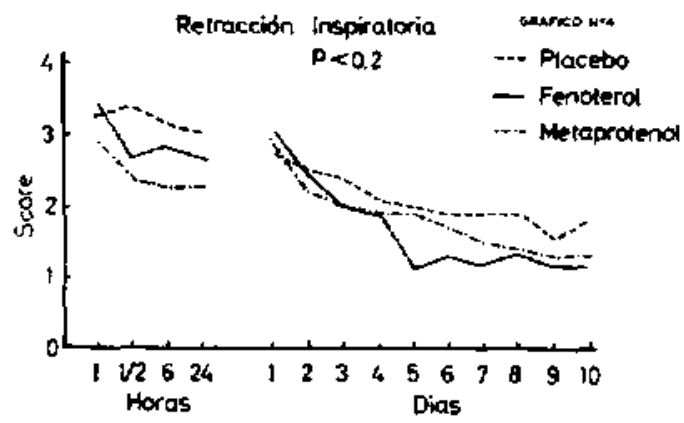

Con respecto a la frecuencia respiratoria, se encontró una diferencia estadísticamente significativa $(P<0.01)$ entre la curva de descenso con Fenoterol y Metaproterenol, en relación al placebo.

En la frecuencia cardiaca se encontró también una diferencia significativa $(P<0.02)$ en la curva de descenso con Fenoterol con respecto al placebo y al Metaproterenol.

En el análisis de los parámetros más subjetivos, como espiración prolongada, retracción inspiratoria, sibilancias y estertores húmedos, no se demostró diferencia significativa entre el grupo con broncodilatadores y el grupo control. (Ver gráficos $3,4,5$ y 6 .)

Se analizó en forma separada la firecuencia respiratoria en los distintos grupos etarios. (Gráficos 7 y 8 .)

Grúfices

ENSAYO CLINICOCON

BRONCODILATADORES EN BRONQUITIS

OBSTRUCTIVA DEL LACTANTE $Y$ PRE-ESCOLAR MENOR.

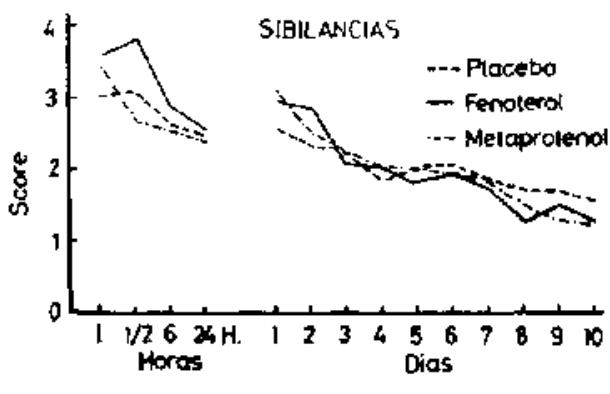

Gráfico o

ENSAYO CLINICO CON

BRONCODILATADORES EN BRONQUITIS OBSTHUCTIVA DEL LACTANTE Y PRE-ESCOLAR MENOR.

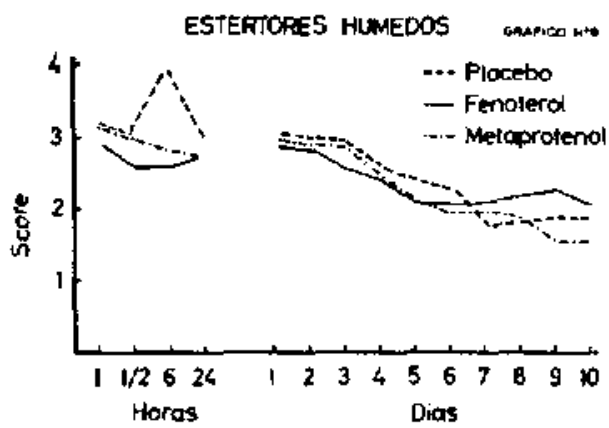




\section{ENSAYO CLINICO CON \\ BRONCODILATADORES EN BRONQUITIS \\ OBSTRUCTIVA DEL LACTANTE Y PFE-ESCOLAR MENOR.}

FRECUENCIA RESPIRATORIA

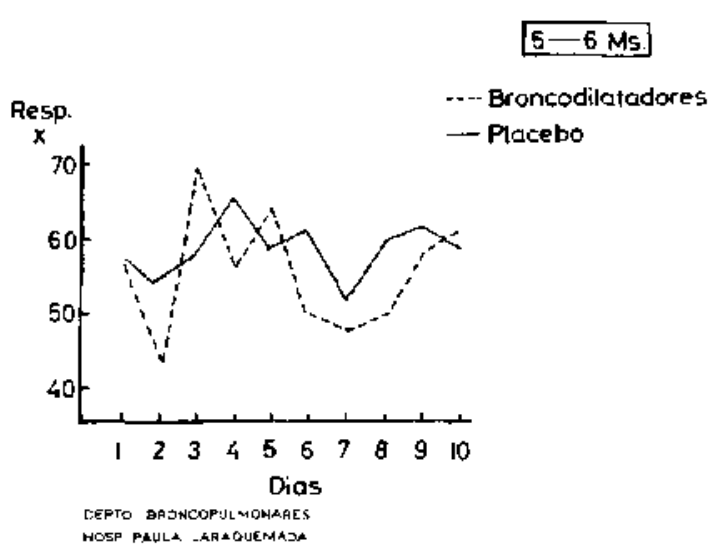

Grálico 8
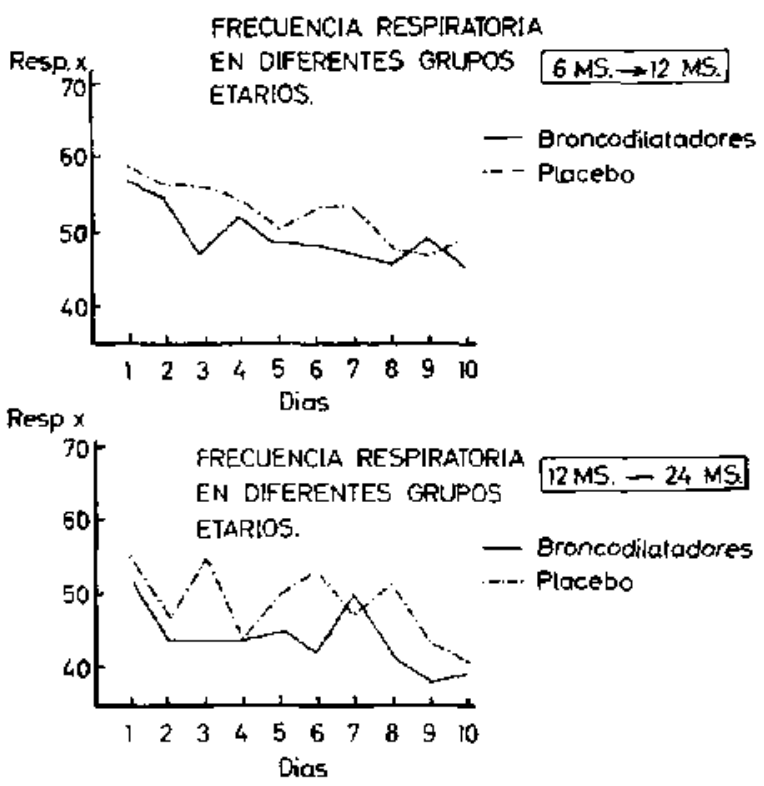

Se encontró que de acuerdo a lo señalado en là literatura, ${ }^{5}$ en el gnupo de menor edad las curvas de descenso de la frecuencia respiratoria eran similares de los tratados con broncodilatadores a los del grupo control. En cambio, en el grupo de mayor edad, sobre los 12 meses, hubo cierta diferen- cia entre los tratados con broncodilatadores y los que recibieron placebo.

Por tratarse de un pequeño grupo en este último análisis, no se hizo estudio estadistico.

\section{DISCUSION}

El concepto de que los broncodilatadores, incluso los estimulantes Beta $\mathbf{2}$ adrenérgicos, sean ineficaces para el manejo terapéutico del Síndrome Bronguial Obstnuctivo del lactante, no estaria bien dilucidado.

En esta experiencia se pudo apreciar cierta mejoría clínica, traducida fundamentalmente por la disminución de la frecuencia respiratoria y cardíaca (esta última sólo con Fenoterol), en pacientes con bronquitis obstructiva, tratados con Beta 2 agonistas. La interpretación de este hecho, en relación a una eventual disminución de la resistencia de la via aérea, debería ser confirmada con estudios funcionales, mediante exámenes pletismográficos u oscilométricos.

Sin embargo, ta utilidad de los Beta 2 agonistas no reside sólo en el efecto broncodilatador, sino también en la acción estimulante de la función mucociliar, a través de modificaciones fisicas de las secreciones del äbol respiratorio, provocadas por estos agentes, facilitando con ello la motilidad ciliar. Esto debemos considerarlo como factor importante en la desobstrucción de la via aérea del niño menor, en el que predomina la distunción mucociliar provocada por alteraciones inflamatorias.

Debemos recordar, al respecto, que un $70 \%$ de nuestros enfermos presentó modificaciones radiológicas pulmonares, compatibles con neumonitis, cuya asociación a componentes inflamatorios bronquiales suele ser casi constante.

Un factor que podria contribuir a la falta de respuesta a estas drogas, en el niño menor, seria su escasa capacidad de aspiración del tármaco por via inhalatoria, que no garantizaria su acción tópica en la fase inicial, siendo insignificante la absorciôn bucofaríngea del aerosol para obtener niveles útiles. Por este motivo se prefinió la terapia asociada a la administración oral, que de todas maneras demostró un efecto limitado.

Consideramos que la evaluación de los otros parámetros clínicos, registrada en el score, es dificil de objetivar, pero creemos que a través de su análisis, que no demuestra diferencias significativas con el grupo control, podría encontrarse cierta 
concordancia con las escasas comunicaciones ${ }^{\text {-13it }}$ que destacan el efecto limitado de las drogas bagonistas, objetivado mediante estudios funcionales.

El efecto más favorable del tratamiento representado por la disminución de la frecuencia respiratoria fụe más evidente en los mayores de 12 meses, lo que puede atribuirse, en parte, a las condiciones anatonofuncionales del lactante menor, que explican el aumento de la resistencia al thajo aéreo $y$, por otra parte, al mayor desamollo muscular bronquial en el de mayor edad, condi. cionante del componente broncos pástico como factor olsstructivo.

Para sintetizar, podríamos concl ${ }_{11} i \mathrm{r}$ que si bien los agentes Beta 2 agonistas han demostrado un efecto limitado en este material de enfermos, no dejan de representar alguna utilidast en el manejo terapéutico del Sindrome Bronquind Obstructivo del lactante, que es mais evidente en el mayor de 1 ano, y que, probablemente, se relaciona en parte con la desobstrucción lograda a trates de su inthiencia sobre la disfuncion mucociliar.

\section{RESUMEN}

Con el objeto de evaluar la respuesta con broncodilatadores en niños menores con bronquitis obstructiva, se realizó un estudio dínico con $\mathrm{Fe}$ noterol y Metaproterenol, comparíndolo con un grupo control.

El an álisis estadístico de cada parametro clínico demostró diferencia significativa solamente en relación al descenso de la frecuencia respiratoria, con el uso de estimulantes beta-adrenérgicos con respecto a los controles. En los demás parámetros no se demostró diferencia estadísticamente significativa entre el grupo con broncodilatadores y el grupo control.

Por lo tanto, este estudio corrobora la utilidad limitada del uso de broncodilatadores en bronquitis obstructiva, traducida en una disminución de la frecuencia respiratoria, que indirectamente demuestra una disminución de la resistencia al flujo aéreo, hecho que debería ser confirmado con pletisnóggrafo.

\section{REFERENCIAS}

'Avner. "Broncudilatadores beta-adrenérgicos", Ped. Clin. of N.A. $22 ; 129,1975$.

"Boffus, W. P. "Asma in infancy". Ped. Clin. of X.A. 6, fix 3; 1959.

3F. Enogar, G. Sholby. D. Ruer. "Terapéutica de la branquitis espastica en el lactante y niño menor con un nuevo espasmolitico bronquial". Deutches Medzen Joumid 20, 12; 1964.

"Adans Wanner. "Cliriteal aspects of Mucuciliary transport". AM1. Res, of Respiratory Dis, 116: 73, 1977 .

5 Lenny and Mitner. "At what agre do Beta Adrenergics work". Arch. Dis, of Child. 53, 532, 1978 .

"Leary and Miner. "Recurrent wheezing Bronchitis is Prescholus Child". Arch. Dis. of Chukl. 53, 468; 1978.

7 Fred Leffer M.D. "Management of acute severe asma", J. M. Ped. Jourrial 1978, Vol. N."1.

"Pheelan tand Williums. "Study of Respiratory Function in Indants with Recurent Asmatic Bronchitis:. Aust. Ped. J. 5, 187; 1969.

"Pheekn and Willians. "Simpatominnetic drugs in aciute Bronchiolitis". Pediatrics 44, 493, 1969.

I" Phetan and Williams. "The Disturtunce of Ventilation in ucrte viral Pronchiolitis". Aust. Ped. 4. 96: 1968.

11 Radford. "Effects of Salbutomol on Wheezing Branchitis". Arch. Dis, of Child, 49, 243; 1974.

12 Rumer and Miner. "Effects of Broncodilators on Respiratury Resistance in Lnfancy and young child with Bronchitis and Wheezing Bronchitis". Arch. Dis. of Child, 50, 719; 1975.

1:3 Whot and Srigal. "Respiratory Resistance in healthy inlants with Bronchiolitis". Ped. 53, 445, April 1969. 